\title{
Association of TP53 Mutations with Response to Anlotinib Treatment in Advanced Non-Small Cell Lung Cancer
}

This article was published in the following Dove Press journal: OncoTargets and Therapy

\author{
Shencun Fang' \\ Wanwan Cheng' \\ Meiling Zhang ${ }^{2}$ \\ Rusong Yang ${ }^{3}$ \\ 'Department of Respiratory Medicine, \\ The Affiliated Brain Hospital of Nanjing \\ Medical University, Nanjing, Jiangsu, \\ People's Republic of China; ${ }^{2}$ Department \\ of Oncology, The First Affiliated Hospital \\ of Nanjing Medical University, Nanjing, \\ Jiangsu, People's Republic of China; \\ ${ }^{3}$ Department of Thoracic Surgery, The \\ Affiliated Brain Hospital of Nanjing \\ Medical University, Nanjing, Jiangsu, \\ People's Republic of China
}

\begin{abstract}
Multitargeted antiangiogenic drugs have demonstrated significant antitumor activity against a variety of solid tumors. Anlotinib, a novel oral multitargeted antiangiogenic tyrosine kinase inhibitor, was approved as a third-line treatment for advanced NSCLC in China. However, predictive biomarkers are currently insufficient and are urgently required. Herein, we report three pre-treated cases of advanced NSCLC with TP53 mutations, wherein these patients showed partial response to anlotinib. Moreover, the three patients have achieved a progression-free survival of $8,6.5$, and 5 months, respectively. The main toxicities were hypertension, hand-foot syndrome and fatigue. In conclusion, TP53 mutations may represent a biomarker for predicting salutary effects of anlotinib.
\end{abstract}

Keywords: non-small cell lung cancer, angiogenesis, anlotinib, TP53

\section{Introduction}

Non-small cell lung cancer (NSCLC), accounting for $85 \%$ of all lung cancers, is the most common cause of cancer-related mortality. ${ }^{1}$ The platinum-based doublet chemotherapy had long been the standard first-line treatment for advanced NSCLC, with a median overall survival (OS) of 8-10 months. ${ }^{2}$ Meanwhile, multitargeted antiangiogenic drugs have also demonstrated encouraging antitumor activity in a variety of malignancies. For example, pazopanib and sorafenib have been used in the treatment of advanced renal cell carcinoma and hepatocellular carcinoma, respectively. ${ }^{3,4}$ Nevertheless, a substantial proportion of patients failed to experience benefit. Given that antiangiogenic drugs may be associated with significant toxicity as well as high cost of treatment, it would be ideal to identify patients who are likely to benefit from such treatment before initiating therapy.

Anlotinib, a novel oral multitargeted antiangiogenic tyrosine kinase inhibitor, was approved as a third-line treatment for refractory advanced NSCLC by the China Food and Drug Administration in 2018..$^{5}$ Recently, a number of studies have introduced some predictors of response to anlotinib treatment. ${ }^{6,8}$ Unfortunately, to date, no predictors available has yet been identified to predict the response to antiangiogenic drug anlotinib.

Recently, there is emerging evidence to support that malignant tumors with mutations in the TP53 gene have better response to antiangiogenic drugs than TP53 wild-type. Wheler et $\mathrm{al}^{9}$ found that the application of vascular endothelial growth factor receptor (VEGFR) inhibitors is an independent factor significantly associated
Department of Oncology, The First Affiliated Hospital of Nanjing Medical University, 300 Guangzhou Road, Nanjing, Jiangsu, People's Republic of China Email zml_1984I025@I63.com 
with favorable prognosis in patients with TP53-mutant cancers. Additionally, a retrospective review demonstrated that response to multitargeted antiangiogenic drug pazopanib can be predicted by TP53 mutational status in advanced sarcomas. ${ }^{10}$ However, there is no report focusing on the relationship between anlotinib and TP53 mutation in in patients with NSCLC. Herein, we report three cases of advanced NSCLC with TP53 mutation, wherein the patients showed partial response (PR) to anlotinib.

\section{Case Report}

\section{Case I}

A 76-year-old female presented with persistent cough and chest tightness with a performance status (PS) of 1 . Computed tomography (CT) scan showed a left inferior pulmonary nodule, multiple enlarged mediastinal lymph nodes, and right pleural effusions. The percutaneous pulmonary biopsy indicated pulmonary adenocarcinoma. A tissue-based NGS assay was performed, showing a MAP2K1 deletion mutation, along with TP53 p.S183X on exon 5 and TP53 p. S241F on exon 7 mutations (Table 1). As first-line chemotherapy, the regimen of "pemetrexed plus cisplatin" was administered for two cycles and had a response of progressive disease (PD). The patient's symptoms further deteriorated with the aggravated PS. At this time, systemic chemotherapy was not the preferred second-line treatment due to her poor PS and advanced age. Then, she received anlotinib treatment $(12 \mathrm{mg}$, D1-D14, Q3W) and partial response (PR) was detected after 1 month of treatment (Figure 1). Moreover, the dyspnea obviously diminished along with the improved PS within 1 week. Progression-free survival (PFS) after anlotinib treatment was 8 months. The only toxicity was grade 2 hypertension, which was well managed without dose adjustment.

\section{Case 2}

A 72-year-old woman with a history of coronary heart disease, atrial fibrillation, and severe cardiac insufficiency for more than 10 years was admitted to our department because of a 2-week history of chest tightness. Chest CT revealed that there was a lesion at the right lower lobe, with carcinomatous lymphangitis in the right lung as well as multiple lymphadenopathies and right pleural effusion. Lung adenocarcinoma was diagnosed on pleural fluid cytology. Given symptomatic pulmonary disease and poor PS, pemetrexed was administered without waiting for mutation status. Unfortunately, the symptoms of chest distress worsened and pleural effusion molecular test result was available, which revealed the EGFR L858R and EGFR T790M activating mutations. Osimertinib was then prescribed and PR was obtained. However, after 3 months of treatment, her symptoms dramatically deteriorated with the disease progressed, resulting in PS 3.

Table I Baseline Characteristics of Patients

\begin{tabular}{|c|c|c|c|}
\hline Characteristics & Case I & Case 2 & Case 3 \\
\hline Age (years) & 76 & 72 & 66 \\
\hline Sex & Female & Female & Female \\
\hline Smoking & No & No & No \\
\hline Pathological type & Adenocarcinoma & Adenocarcinoma & Adenocarcinoma \\
\hline PS & 2 & 3 & 2 \\
\hline TP53 mutation & Exon 5 and 7 & Exon 5 & Exon 9 \\
\hline \multicolumn{4}{|l|}{ Therapy (response) } \\
\hline First line & Pemetrexed/cisplatin (PD) & Pemetrexed (PD) & Pemetrexed/cisplatin (SD) \\
\hline Second line & Anlotinib (PR) & Osimertinib (PR) & Afatinib (PD) \\
\hline Third line & & Anlotinib (PR) & Anlotinib (PR) \\
\hline PFS (months) & 8 & 6.5 & 5 \\
\hline \multicolumn{4}{|l|}{ Adverse events } \\
\hline Hypertension & Grade 2 & Grade I & Grade 2 \\
\hline Hand-foot syndrome & & Grade I & Grade I \\
\hline Fatigue & & Grade I & Grade I \\
\hline
\end{tabular}



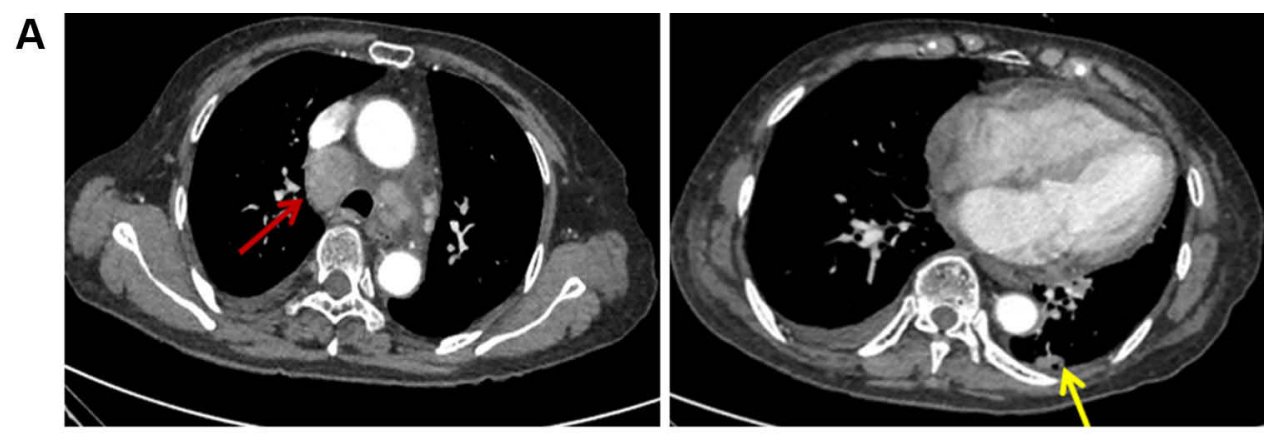

B
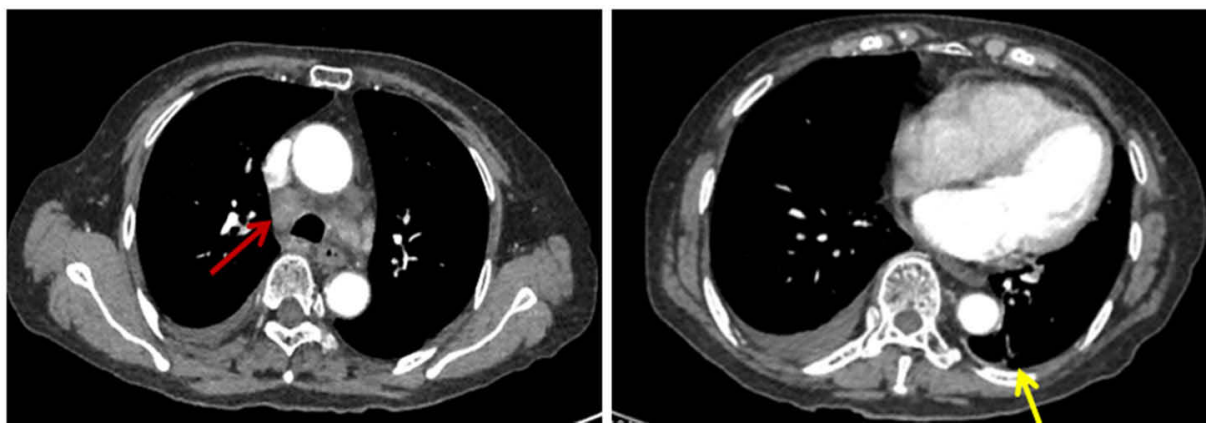

Figure I Case I: Computed tomography shows a left inferior pulmonary nodule and multiple enlarged mediastinal lymph nodes (A) Before anlotinib treatment and (B) After anlotinib treatment was given for 6 weeks. Red arrows: metastatic lymph nodes. Yellow arrows: primary lung tumor.

A broad NGS test from a repeat biopsy specimen was performed, showing a TP53 p.R175H on exon 5 mutation (Table 1). Therefore, treatment with anlotinib (12mg, D1D14, Q3W) was administrated as a third-line treatment. The primary tumor, metastases, and pleural effusion were significantly shrunk with PS improvement after 1 month of anlotinib treatment (Figure 2). She has achieved a PFS of 6.5 months. The side effects during anlotinib treatment were controllable hypertension (grade 1), hand-foot syndrome (grade 1), and fatigue (grade 1).

A
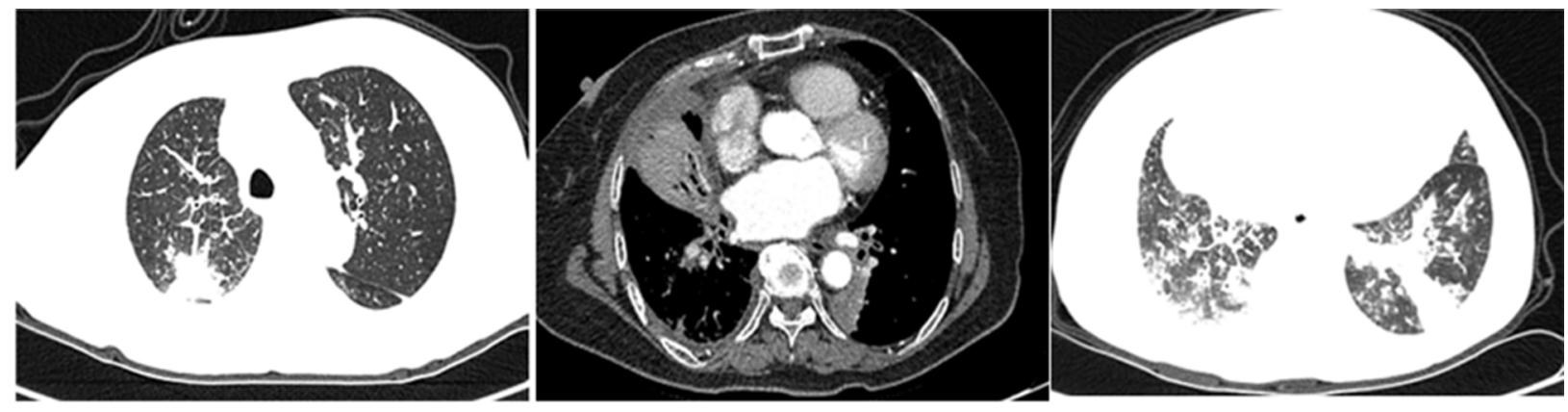

B
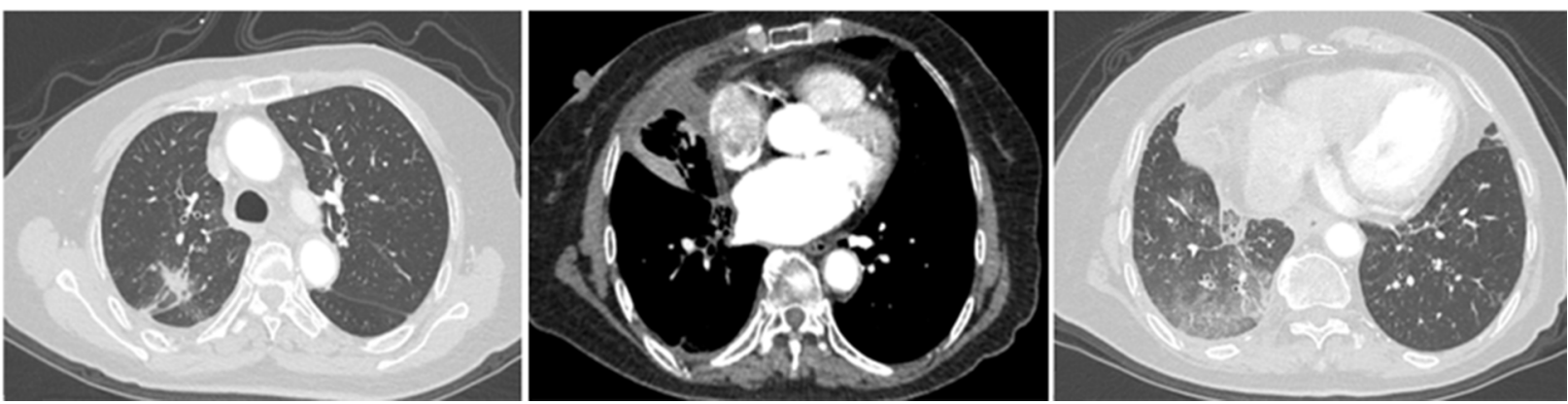

Figure 2 Case 2: Computed tomography shows the primary tumor in the right lower lung and metastases in the left and right upper lung. (A) Before anlotinib treatment and (B) After anlotinib treatment was given for 4 weeks. 

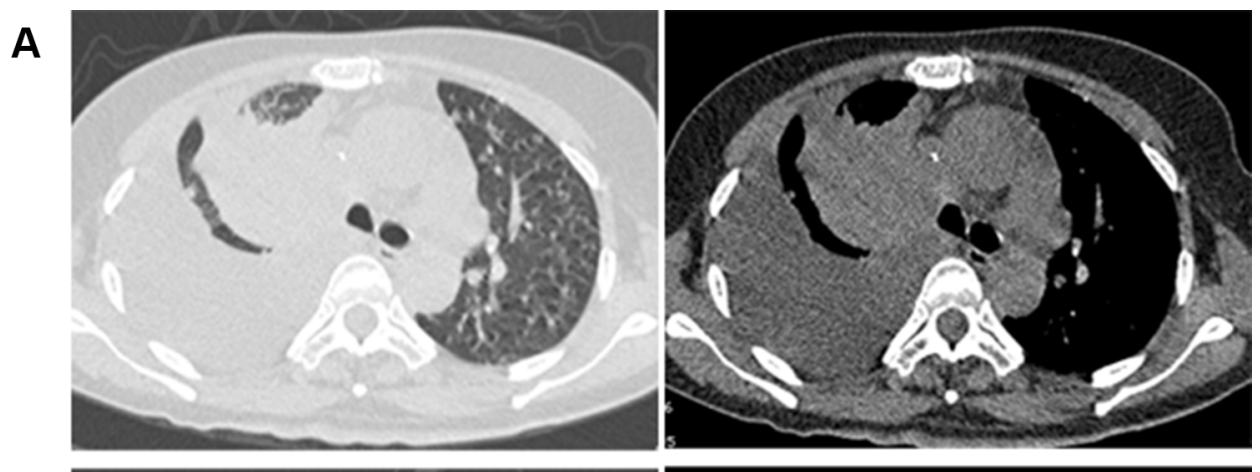

B
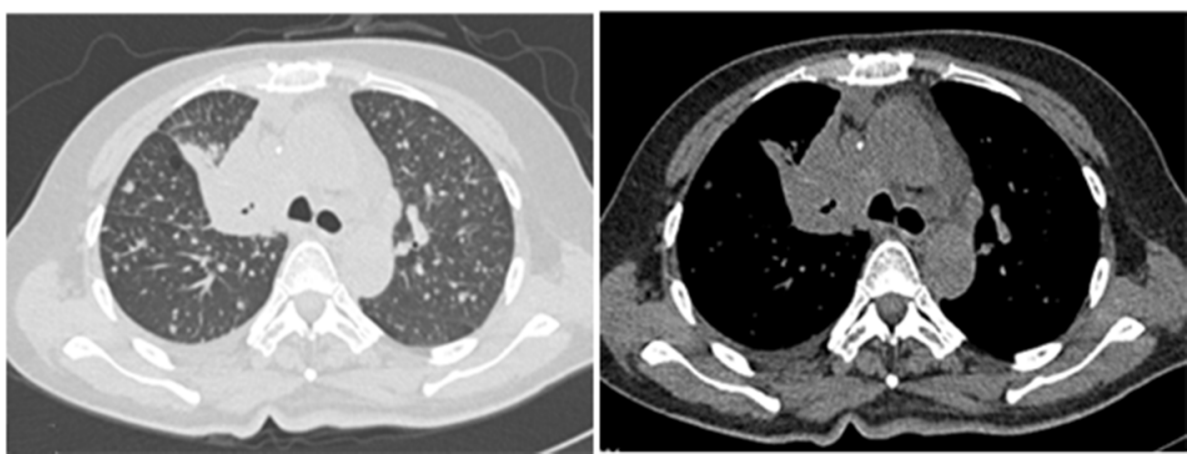

Figure 3 Case 3: Computed tomography shows a mass in the right upper lobe with right pleural effusion. (A) Before anlotinib treatment and (B) After anlotinib treatment was given for I week.

\section{Case 3}

A 66-year-old, non-smoking woman presented with low back pain for 1 month. Chest CT revealed a $5.7 \mathrm{~cm} \times 4.4 \mathrm{~cm}$ mass in the right upper lobe, with diffuse metastatic nodules in both lungs and moderate right pleural effusion. Magnetic resonance imaging (MRI) of the spine revealed multiple bone metastases of thoracic, lumbar, and sacral vertebrae with compression fractures of the 11th thoracic vertebra (T11). Lung adenocarcinoma was diagnosed on lung biopsy and molecular test results showed ERBB2 mutation along with TP53 K320fs on exon 9 mutation (Table 1). The regimen of "pemetrexed plus cisplatin" was administered as a first-line treatment and had a response of stable disease (SD). Due to the worsening of lumbago and poor PS, The patient promptly started on combination therapy with local radiotherapy and afatinib. Despite this, PD in multiple lung lesions was diagnosed after one cycle. The patient received anlotinib(12mg, D1D14, Q3W) as a third-line treatment. By 1 week after the start of anlotinib, his symptoms gradually improved along with a noticeable reduction in tumor size (Figure 3), resulting in better PS. PFS after anlotinib treatment was 5 months. The main toxicities were hypertension (grade 2), hand-foot syndrome (grade 1), and fatigue (grade 1).

\section{Discussion}

TP53 as the guardian of the human genome is a multifunctional tumor suppressor gene and plays multiple crucial roles in cancer progression. TP53 mutations are the most frequent genomic abnormality across cancers, with about $40-50 \%$ of diverse tumors harboring these alterations. ${ }^{11}$ To date, there is no effective therapeutic strategy available that specifically targets TP53 mutations, though several approaches are currently under evaluation in clinical trials.

Various TP53 mutations have been identified to be involved in the process of neovascularization associated with increased VEGF expression, ${ }^{12}$ which promotes tumor growth and metastasis, leading to the therapeutic benefits of antiangiogenic VEGF inhibitors in patients with p53 mutant malignancies. ${ }^{13}$ There is mounting clinical evidence that demonstrates a favorable response to antiangiogenic drugs in patients with p53 mutations. For example, pazopanib, an orally multitargeted tyrosine kinase inhibitor, mainly targets VEGFR-1 to 3 . Fu et al ${ }^{13}$ carried out a Phase I trial of pazopanib in combination with the histone deacetylase inhibitor, vorinostat, in patients with advanced solid malignancies. The study showed that patients with a TP53 mutation had significantly higher response rates and a longer median OS, than those without 
TP53 mutation. A recent retrospective study of patients with advanced sarcomas also demonstrated that VEGFR inhibition appeared to be more effective in TP53 mutant versus TP53 WT tumors. ${ }^{10}$ Additionally, a prospective study was conducted to analyze the clinical correlates of TP53 status in 188 patients with advanced cancers. ${ }^{9}$ The results showed that patients with TP53 mutation, rather than TP53 wild-type, had favorable outcomes when treated with VEGF/VEGFR inhibitors. Therefore, the abovementioned results were consistent with the observations of this study, which supports our findings that patients harboring TP53 mutation are more likely to respond to VEGF/VEGFR inhibitors.

Similar to the above multitargeted antiangiogenic drugs, anlotinib also effectively inhibits tumor angiogenesis and growth mainly through suppressing the signal pathway mediated by VEGFR-1 to 3 and fibroblast growth factor receptor (FGFR)- 1 to $4 .^{14}$ The ALTER0303 study has demonstrated that anlotinib therapy was beneficial in prolonging median progression-free survival (PFS) and median overall survival (OS) in heavily pre-treated NSCLC patients. ${ }^{5}$ However, no research investigating the relationship between TP53 mutation and response to anlotinib has been reported, to the best of our knowledge. But it is noteworthy that all of the three patients mentioned above treated with anlotinib who received PR, harbored TP53 mutations. Recently, a case report described favorable responses to anlotinib in a pulmonary artery sarcoma patient harboring TP53 mutation. ${ }^{15}$ Furthermore, TP53 mutations, lacking most of the DNA-binding domain and deficient in transactivation function, leads to loss of function of TP53 in thyroid cancer cells, which can be reactivated by anlotinib. ${ }^{16}$ Based on these preliminary data, we speculated that TP53 mutations might be a biomarker to predict the beneficial effect of anlotinib. A larger, randomized control-based trial will be needed to confirm the results of the present study.

\section{Ethics Approval and Consent for Publication}

This study was approved by the research ethics committee of the Affiliated Brain Hospital of Nanjing Medical University (Nanjing, China; Approval No. of Ethics Committee: 2019-KY023-02), and written informed consent for publication of the clinical details and images was obtained from the patients. Institutional approval was not required to publish this manuscript.

\section{Acknowledgments}

The authors thank the patients for their participation in the study and agreeing to the publishing of the report.

\section{Author Contributions}

All authors contributed toward data analysis, drafting, and critically revising the paper, gave final approval of the version to be published, and agree to be accountable for all aspects of the work.

\section{Funding}

This study was supported by the CSCO Oncology Research Foundation (Y-Q201802-018) and Six One Projects (LGY2019006).

\section{Disclosure}

The authors have no conflicts of interest to disclose related to the current manuscript.

\section{References}

1. Bray F, Ferlay J, Soerjomataram I, Siegel RL, Torre LA, Jemal A. Global cancer statistics 2018: GLOBOCAN estimates of incidence and mortality worldwide for 36 cancers in 185 countries. CA Cancer J Clin. 2018;68(6):394-424. doi:10.3322/caac.21492

2. Goldstraw P, Chansky K, Crowley J, et al. The IASLC lung cancer staging project: proposals for revision of the TNM stage groupings in the forthcoming (eighth) edition of the TNM classification for lung cancer. $J$ Thorac Oncol. 2016;11(1):39-51. doi:10.1016/j. jtho.2015.09.009

3. Motzer RJ, Hutson TE, Tomczak P, et al. Sunitinib versus interferon alfa in metastatic renal-cell carcinoma. $N$ Engl $J$ Med. 2007;356 (2):115-124. doi:10.1056/NEJMoa065044

4. Motzer RJ, Haas NB, Donskov F, et al. Randomized phase III trial of adjuvant pazopanib versus placebo after nephrectomy in patients with localized or locally advanced renal cell carcinoma. J Clin Oncol. 2017;35(35):3916-3923. doi:10.1200/JCO.2017.73.5324

5. Han B, Li K, Wang Q, et al. Effect of anlotinib as a third-line or further treatment on overall survival of patients with advanced non-small cell lung cancer: the ALTER 0303 phase 3 randomized clinical trial. JAMA oncol. 2018;4(11):1569-1575. doi:10.1001/ jamaoncol.2018.3039

6. Lu J, Zhong H, Chu T, et al. Role of anlotinib-induced CCL2 decrease in anti-angiogenesis and response prediction for nonsmall cell lung cancer therapy. Eur Respir J. 2019;53(3). doi:10.1183/ 13993003.01562-2018

7. Wang J, Zhao Y, Wang Q, et al. Prognostic factors of refractory NSCLC patients receiving anlotinib hydrochloride as the third- or further-line treatment. Cancer Biol Med. 2018;15(4):443-451. doi:10.20892/j.issn.2095-3941.2018.0158

8. Wang L, He Z, Yang S, et al. The impact of previous therapy strategy on the efficiency of anlotinib hydrochloride as a third-line treatment on patients with advanced non-small cell lung cancer (NSCLC): a subgroup analysis of ALTER0303 trial. Transl Lung Cancer Res. 2019;8(5):575-583. doi:10.21037/tlcr.2019.09.21

9. Wheler JJ, Janku F, Naing A, et al. TP53 alterations correlate with response to VEGF/VEGFR inhibitors: implications for targeted therapeutics. Mol Cancer Ther. 2016;15(10):2475-2485. doi:10.1158/ 1535-7163.MCT-16-0196 
10. Koehler K, Liebner D, Chen JL. TP53 mutational status is predictive of pazopanib response in advanced sarcomas. Ann Oncol. 2016;27 (3):539-543. doi:10.1093/annonc/mdv598

11. Levine AJ, Oren M. The first 30 years of p53: growing ever more complex. Nature Reviews Cancer. 2009;9(10):749-758. doi:10.1038/ nrc2723

12. Li AM, Boichard A, Kurzrock R. Mutated TP53 is a marker of increased VEGF expression: analysis of 7525 pan-cancer tissues. Cancer Biol Ther. 2020:1-6.

13. Fu S, Hou MM, Naing A, et al. Phase I study of pazopanib and vorinostat: a therapeutic approach for inhibiting mutant p53-mediated angiogenesis and facilitating mutant p53 degradation. Ann Oncol. 2015;26(5):1012-1018. doi:10.1093/annonc/mdv066
14. Sun Y, Niu W, Du F, et al. Safety, pharmacokinetics, and antitumor properties of anlotinib, an oral multi-target tyrosine kinase inhibitor, in patients with advanced refractory solid tumors. J Hematol Oncol. 2016;9(1):105. doi:10.1186/s13045-016-0332-8

15. Wu Y, Huang J, Wang Q, et al. Whole-exome sequencing insights into pulmonary artery sarcoma mimicking pulmonary embolism: a case report and review. Onco Targets Ther. 2019;12:6227-6235. doi:10.2147/OTT.S212416

16. Ruan X, Shi X, Dong Q, et al. Antitumor effects of anlotinib in thyroid cancer. Endocr Relat Cancer. 2019;26(1):153-164. doi:10.1530/ERC-17-0558

\section{Publish your work in this journal}

OncoTargets and Therapy is an international, peer-reviewed, open access journal focusing on the pathological basis of all cancers, potential targets for therapy and treatment protocols employed to improve the management of cancer patients. The journal also focuses on the impact of management programs and new therapeutic

Submit your manuscript here: https://www.dovepress.com/oncotargets-and-therapy-journal agents and protocols on patient perspectives such as quality of life, adherence and satisfaction. The manuscript management system is completely online and includes a very quick and fair peer-review system, which is all easy to use. Visit http://www.dovepress.com/ testimonials.php to read real quotes from published authors. 